\title{
1 Variation in reaction norms: statistical considerations and biological

$5{ }^{1}$ School of Biology, University of St Andrews

$6 \quad{ }^{2}$ Department of Animal Ecology, VU University Amsterdam

7

${ }^{3}$ contact

email: michael.morrissey@st-andrews.ac.uk

phone: +44 (0) 1334463738

fax: $\quad+44(0) 1334463366$

post: Dyers Brae House

School of Biology, University of St Andrews

St Andrews, Fife, UK, KY16 9TH 


\section{Abstract}

9 Analysis of reaction norms, the functions by which the phenotype produced by a given geno-

10 type depends on the environment, is critical to studying many aspects of phenotypic evo-

11 lution. Different techniques are available for quantifying different aspects of reaction norm

12 variation. We examine what biological inferences can be drawn from some of the more readily-

13 applicable analyses for studying reaction norms. We adopt a strongly biologically-motivated

14 view, but draw on statistical theory to highlight strengths and drawbacks of different tech-

15 niques. In particular, consideration of some formal statistical theory leads to revision of 16 some recently, and forcefully, advocated opinions on reaction norm analysis. We clarify what

17 simple analysis of the slope between mean phenotype in two environments can tell us about 18 reaction norms, explore the conditions under which polynomial regression can provide ro19 bust inferences about reaction norm shape, and explore how different existing approaches 20 may be used to draw inferences about variation in reaction norm shape. We show how mixed 21 model-based approaches can provide more robust inferences than more commonly-used multi22 step statistical approaches, and derive new metrics of the relative importance of variation in 23 reaction norm intercepts, slopes, and curvatures. 


\section{Introduction}

25 Characterising the functions describing the dependence upon the environment of phenotypes

26 generated by specific genotypes is critical to understanding many aspects of evolution. These

27 functions, termed reaction norms (Schmalhausen, 1949; Woltereck, 1909), are therefore the

28 subject of a great deal of interest from evolutionary biologists (Gupta and Lewontin, 1982;

29 Scheiner, 1993; West-Eberhard, 2003). For example, characterisations of reaction norms can

30 be important for understanding how populations will respond to changing environments,

31 and so the extent to which non-evolutionary plastic responses and adaptive evolutionary

32 change can allow populations to persist (Chevin et al., 2010; Ghalambor et al., 2007). In

33 microevolutionary studies, we may often be interested both in the mean reaction norm of

34 populations, and also in variation in reaction norms within populations (Nussey et al., 2005).

35 Assessment of variation in reaction norms can in principle inform us of how traits will evolve

36 in response to selection across a range of environments (Kirkpatrick et al., 1990; Scheiner

37 and Callahan, 1999).

38 The true shapes of reaction norms are potentially complex, and any empirical analysis 39 will require a model of reaction norms (DeWitt and Scheiner, 2004; Gavrilets and Scheiner, 40 1993). Two general principles of models will hold true for the analysis of reaction norms.

41 First, models of reaction norms will typically be simpler than the true (unknown) functions

42 themselves. This simplification is not a weakness of model-based approaches, but in fact is

43 key to generating tractable inferences. Second, simple models may also have properties that

44 do not reflect, or only poorly reflect, some properties of true reaction norms. These two

45 general principles will invariably apply both to model-based inferences of specific reaction

46 norms (e.g., the average response of a genotype or population to an environmental variable),

47 and to inferences about variation in reaction norms (e.g., inferences of the amount of variation

48 in say, the steepness of reaction norms among different genotypes in a population).

49 The primary goal of this paper is to examine how some of the most readily-applicable

50 statistical models of reaction norms can be used to make robust inferences about properties 
51 of reaction norms. As examples of such reaction norm properties, we consider inference both 52 of properties of reaction norms of individual genotypes (or other genetic groupings such as 53 populations; e.g., focusing on their slopes, or the locations of their maxima), and properties of 54 families of reaction norms (e.g., variation in slopes, or variation in the locations of maxima). 55 The primary focus is on biological inference, but we draw extensively on the statistical 56 theory underlying different potential analytical approaches to studying reaction norms. In 57 some cases, we expand basic theory about regression analysis to yield new insights about 58 how specific, biologically-motivated, regression analyses may behave. We discuss biological 59 inference of properties of reaction norms in general, but we also specifically focus on on some 60 recent claims that have been made about the efficacy of different approaches.

61 Polynomial regression, and especially quadratic regression, is potentially very useful for 62 characterising reaction norms, and several authors have investigated theoretical and empir63 ical properties of reaction norms using such functions as theoretical and statistical models 64 (e.g., Delpuech et al. 1995; Gavrilets and Scheiner 1993). Two recent very firm claims about 65 analysis of reaction norms with polynomial functions are: (1) that the slope of a line con66 necting mean phenotype in two environments is generally misleading about the form of a 67 reaction norm (Rocha and Klaczko, 2012); and (2) that quantities derivable from polynomial 68 regressions, such as the slope at any point, or measures of overall curvature, provide robust 69 inference of reaction norms (Rocha and Klaczko, 2014). We show analytically, and with 70 numerical examples, that neither of these assertions is generally true. Nonetheless, we agree 71 that polynomial regression, perhaps especially quadratic regression, may be very useful for 72 biological studies of reaction norms. However, polynomial regression will be most useful if 73 applied with a somewhat more nuanced understanding of its strengths and limitations.

74 We also contrast two approaches to characterising variation in reaction norms. By "char75 acterising variation", we refer to situations where we are not necessarily interested in specific 76 reaction norms, nor in comparisons of properties of two or few specific reaction norms, but 77 rather where we seek to assess variation in populations for aspects of reaction norms. For 
78 example, we may be interested in how much variation in average slope, relative to variation 79 in mean values, occurs among the reaction norms of the genotypes segregating within some 80 population, or among populations within a species. There are two basic approaches in use 81 to quantifying such variation. In the first procedure, two steps are employed. First, data 82 from each genetic group (individual, genotype, inbred line, etc.) are subjected to statistical 83 analysis, for example, to regression analyses to determine slopes, as well as calculations of 84 line-specific means across environments. In the second step of the first approach, summary 85 statistics are calculated at the population level, providing, for example, measures of variance 86 in the means and slopes estimated in the first step. In the second type of approach, mixed 87 models, in particular, random regression mixed models, may be used to directly estimate 88 variance in reaction norm parameters. We show analytically how the two-step approach in89 troduces biases into most inferences about variation in reaction norms, and we illustrate the 90 application of random regression mixed models, in detail, with an empirical example. We 91 also derive new measures of variation in phenotype arising from different aspects of reaction 92 norms, and show how these may be particularly useful for answering questions of current 93 interest in reaction norm research.

94 This paper is arrayed in several sections. In each, biologically-relevant results and the 95 more intuitive pieces of statistical theory upon which they rest are presented, while more 96 involved statistical theory is generally relegated to an extensive appendix. First, we consider 97 methods for characterising aspects of individual reaction norms, including the slope between 98 mean phenotype in two environments, and polynomial regression. We then turn our attention 99 to inference of variation in reaction norms. We compare the two-step and mixed model-based 100 approaches, present an empirical example, and derive new measures of variation in reaction 101 norm shape. In the discussion, we recapitulate our major points and address various common 102 threads. 


\section{Slopes between two points}

104 The simplest inference of a reaction norm slope is provided by taking the difference between

105 mean phenotype in two environments, for some unit of biological organisation (clone, genetic

106 line, population, species; see for e.g., Berg et al. 2010; Ellers and Driessen 2011; Fallis et al.

107 2014; Liefting et al. 2009). Divided by the difference between the two environments, the

108 difference in mean phenotype gives an estimate of the average slope of the reaction norm

109 between those two points

$$
s_{a b}=\frac{\bar{z}_{b}-\bar{z}_{a}}{b-a}
$$

110 This simple assessment of reaction norm slope has two important properties. First, it is an

111 unbiased estimator of the average slope of a reaction norm between points $a$ and $b$, weighting 112 all values of the environment between $a$ and $b$ equally. The slope of an arbitrary reaction 113 norm function $E[z \mid x]=f(x)$, where $E[z \mid x]$ is the expected phenotype, $z$, given the value of 114 the environmental variable, $x$, at any given point, and $f^{\prime}(x)$ is its derivative of the function $115 f(x)$, at point $x$. An average over a continuous variable can be obtained by integrating the 116 quantity to be averaged, i.e., $f^{\prime}(x)$, over the range of the predictor variable (the environment, $117 x$ between $a$ and $b$ ), while weighting by the probability density of $x$ (in this case a uniform 118 density between $a$ and $b$, which is $\left.\frac{1}{b-a}\right)$, so

$$
E\left[f^{\prime}(x)\right]=\int_{a}^{b} f^{\prime}(x) \frac{1}{b-a} d x
$$

119 Simplifying this expression using basic algebra and calculus rules gives

$$
E\left[f^{\prime}(x)\right]=\int_{a}^{b} f^{\prime}(x) \frac{1}{b-a} d x=\frac{1}{b-a} \int_{a}^{b} f^{\prime}(x) d x=\frac{1}{b-a}\left[\int f^{\prime}(b)-f^{\prime}(a)\right]=\frac{\bar{z}_{b}-\bar{z}_{a}}{b-a}=s_{a b} .
$$

120 Thus, regardless of the true form of the reaction norm function, i.e., of $f(x)$, the very simple 121 expression in equation 1 gives the average slope of the reaction norm, weighting all values 122 between $a$ an $b$ equally. We will presently see that this ability to recover a major and 123 biologically relevant aspect of a reaction norm is not necessarily a property of other analytical 
124 approaches, including some that have recently been advocated in the literature.

125 Second, the basic experimental design associated with the reaction norm analysis in equa126 tion 1 can be shown to be optimal with respect to minimising statistical error in the inference 127 of the average slope. If a researcher can rear a set number of individual organisms across 128 a range of environments, it may be desirable for different purposes to raise them in two 129 environments, i.e., at $x=a$ and $x=b$, or to divide the total sample size among additional 130 environments between $a$ and $b$.

131 It may initially seem that raising organisms across a number of different environments, 132 calculating the slopes between adjacent environmental treatments, and averaging these slopes, 133 would give a better calculation of average slope over some total range of $x$. This is not the 134 case. The standard error of an estimated reaction norm slope between two points, $s_{a b}$ is

$$
S E\left(s_{a b}\right)=\sqrt{\frac{\Sigma\left(\bar{z}_{a}\right)+\Sigma\left(\bar{z}_{b}\right)}{b-a}},
$$

135 where $\Sigma(\bar{z})$ denotes the sampling variance of an environment-specific estimate of mean pheno136 type, i.e., the squares of the standard errors of the estimated means. The sampling variance 137 of the mean, under normality, is the variance divided by the sample size. The sampling vari138 ance of $s_{a b}$ will be minimised when the quantity $\Sigma\left(\bar{z}_{a}\right)+\Sigma\left(\bar{z}_{b}\right)$ is minimised, and if variances 139 are equal in environments $a$ and $b$, this occurs if the total sample size is divided between the 140 two environments. If variances are not equal in the two environments, a design that increases 141 sample size in the environment with more variance will be optimal for minimising error in $142 s_{a b}$.

143 If, alternatively, there were three environments, say $x_{1}, x_{2}$, and $x_{3}$, the mean phenotype 144 in environment $x_{2}$ would appear in the calculation of $s_{a b}$ for both the intervals between $x_{1}$ 145 and $x_{2}$, and between $x_{2}$ and $x_{3}$. This produces a negative sampling covariance between the 146 two estimates of $s_{a b}$ for adjacent ranges of $x$. Consequently, for the purposes of minimising 147 statistical error a single measure of $s_{a b}$ can give the most powerful possible estimate of the 148 average slope of a reaction norm between points $x=a$ and $x=b$. That this design is optimal 
149 with respect to minimising the sampling variance of the average slope is demonstrated more 150 rigorously in appendix section A.1.

\section{Polynomial regression analysis of reaction norms}

152 Polynomial regressions are proven statistical tools for characterising functions, and have

153 been advocated for analysis of non-linear reaction norms (e.g., Gavrilets and Scheiner 1993;

154 Rocha and Klaczko 2014). Polynomial regressions will typically be least-squares fits of an

155 approximating function to a true reaction norm with an unknown true functional form. Given

156 phenotypic values, $z$, and environmental values, $x$, for units of observation indexed $i$, first-

157 (linear), second- (quadratic), and third-order (cubic) polynomial regressions take the form

$$
y_{i}=a+b_{1} x_{i}+e_{i}
$$

158

$$
y_{i}=a+b_{1} x_{i}+b_{2} x_{i}^{2}+e_{i}
$$

$$
y_{i}=a+b_{1} x_{i}+b_{2} x_{i}^{2}+b_{3} x_{i}^{3}+e_{i}
$$

160 In each fitted regression model, the intercept, $a$, and polynomial regression coefficients, i.e., $b_{1}$,

$161 b_{2}$ and $b_{3}$, will be those that minimise the variance of the residuals $(e)$. Note that the values 162 of the intercept and common coefficients (e.g., $b_{1}$ ) may differ between models of different 163 polynomial order, fitted to the same data.

164 In application of polynomial regression, it is hoped that coefficients of the regression 165 model, or predictions from the fitted model, will reflect biologically relevant aspects of re166 action norms. While polynomial regression may often be pragmatic, the conditions under 167 which coefficients of polynomial regression models will reflect specific, biologically relevant, 168 quantities such as the average slope of curvature are limited. Where there is a very simple 169 and general interpretation of $s_{a b}$ as the average slope of a true arbitrary reaction norm be170 tween the points $x=a$ and $x=b$, the conditions under which a polynomial regression can 
171 provide a similar inference are much more limited. If the environmental variable is normally 172 distributed - both in the data analysed and in the relevant scenario in nature about which 173 we want to draw inferences, then the linear term $\left(b_{1}\right)$ in a first-order quadratic regression 174 model gives the average slope. This can be demonstrated from Stein's lemma (Stein, 1973), 175 where it has been shown that $\sigma(x y)=\sigma^{2}(x) E\left[\frac{\delta y}{\delta x}\right]$ if $x$ is normally distributed, not otherwise. 176 A regression coefficient is the covariance of the predictor and the response, divided by the 177 variance of the predictor, so $b_{1}=\frac{\sigma(x z)}{\sigma^{2}(x)}=E\left[\frac{\delta z}{\delta x}\right]$. The linear term in higher-order polynomial 178 regression models estimates the average slope as well, still requiring normality of the envi179 ronmental variable, and also that the environmental variable has a mean of zero. Similarly, 180 if the environmental covariate is normally-distributed and mean-centred, the quadratic term 181 in a quadratic approximation to the reaction norm is equal to half of the average second 182 derivative of the reaction norm function. These specific properties of quadratic regression 183 analysis, when covariates are normal and mean-centred, underlie regression-based analysis of 184 selection gradients, which are the average first and second (partial) derivatives of (relative 185 fitness) functions as well (Geyer and Shaw, 2010; Lande and Arnold, 1983; Mitchell-Olds and 186 Shaw, 1987).

187 So, there is a condition, namely, normality of the environmental covariate $x$, under which 188 coefficients of polynomial regressions have very general and biologically useful interpreta189 tions. Under normality, the slope and curvature of a polynomial approximation to a reaction 190 norm can reflect the average slope and curvature of the true reaction norm, regardless of the 191 true form of the reaction norm. However, the condition of normality of the environmental 192 variable $(x)$ is patently not met in virtually all studies of reaction norms. Rather, by design, 193 the distribution of the environmental variable(s) are non-normal, being composed of two or 194 more discrete treatments. This distribution for $x$ tends toward a uniform distribution as the 195 number of treatments increases. If the distribution of the environmental variable is not nor196 mal, then the parameters of a polynomial regression have no direct biological interpretation, 197 and no single useful statistical interpretation, other than that they are the parameters that 198 minimise the residual variance. However, parameters of a polynomial regression will provide 
199 insight into aspects of true reaction norms, regardless of the distribution of $x$, providing they 200 are a reasonably good approximation of the true reaction norm.

201 There is a corollary of the principle that the parameters of a polynomial regression need 202 only reflect aspects of a true reaction norm under specific assumptions about the distribu203 tion of the covariate that may appear more biologically important. This is as follows: the 204 parameters of a polynomial approximation to a reaction norm are not just functions of the 205 true reaction norm; but, they are also determined by the distribution of the environmental 206 variable in any given analysis. Figure 1 shows polynomial approximations to an arbitrary 207 (non-polynomial) function, as well as the differences in polynomial approximations to a re208 action norm that occur as a result of a near-uniform distribution (i.e., many closely-spaced 209 environmental treatments; figure 1a), and a normal distribution (dashed lines in figure 1b), 210 where both distributions have the same mean and variance. The differences in polynomial 211 shape, arising due to only a change in the distribution of the covariate, are substantial. The 212 quadratic approximation is much more steeply peaked when the distribution of $x$ is uniform, 213 rather than normal. The cubic approximation contains a minimum within the range of the 214 covariate for the uniform distribution, but not for the normal distribution. While the near215 uniform covariate distribution (many, closely-spaced environmental treatments) is advocated 216 (Rocha and Klaczko, 2012) and used (e.g., Morin et al. 1999; Pétavy et al. 2001; Rocha et al. 217 2009), in studies of reaction norms, it may often be that extreme environments are relatively 218 rare in nature, and environmental variables may be more normally distributed. Regardless 219 of specific distributions and their relevance in different situations, the fact that polynomial 220 regressions do not reflect only the reaction norm being studied, but also essentially arbitrary 221 features of a study design, should be strong reason for care in their interpretation. In ap222 pendix section A.2, we demonstrate a simple example where the $s_{a b}$ metric can be useful, 223 and slopes of quadratic approximations of reaction norms may be less useful.

224 There are likely many scenarios where polynomial regression will provide pragmatic and 225 useful statistical models for studying reaction norms. While the reaction norm used for 
226 illustration in figure 1 is very plausible - indeed, this sort of functional form appears in 227 many discussions of thermal reaction norms (e.g., Kingsolver et al. 2004) - many studies 228 will not have to contend with the same degree on non-linearity. As a polynomial regression 229 more closely approximates the true function, predictions from the approximation will better 230 reflect aspects of the biology of the reaction norm. The degree of model complexity, i.e., 231 the degree of a polynomial reaction norm, is difficult to determine. Previous discussions of 232 polynomial reaction norms have suggested forward model selection. Such a procedure can be 233 inconsistent, i.e., can fail to converge on the "true" model (in the hypothetical situation where 234 the true model is included in the set of models that is considered), even when arbitrarily large 235 amounts of data are available. We elaborate on this property of forward model selection of 236 polynomial regression functions in appendix section A.3.

\section{Assessing variation in reaction norms}

238 Variation among reaction norms, for example genetic variation among species, families, clones 239 or inbred lines, is often assessed by first calculating metrics such as $s_{a b}$, or by fitting poly240 nomial regression functions, to each genetic unit (e.g., clone, genotype, sibship). In a second 241 step, variances (or other measures of variation) in $s_{a b}$, or of regression parameters, are cal242 culated. This basic procedure will exaggerate apparent levels of variation in any feature of 243 reaction norms, a principle that can be demonstrated with some simple theory about the 244 sampling variance of regression coefficients.

245 The sampling variance of the mean (the intercept in a linear model with a symmetric, 246 mean-centred covariate) is

$$
\Sigma[\bar{z}]=\Sigma[\mu]=\frac{\sigma_{r}^{2}}{n}
$$

247 (the square-root of which is the familiar formula for the standard error of a mean), and the 248 sampling variance of the slopes is

$$
\Sigma\left[b_{1}\right]=\frac{3 \sigma_{r}^{2}}{n r^{2}}
$$


249 A derivation of this expression is given in the appendix, section A.4.

250 What is the significance of these sampling error variances? In the common two-step pro251 cedure, where parameters such as line-specific slopes are first calculated, and then variances 252 (or other summary statistics) of those statistical estimates are subsequently calculated, sta253 tistical noise in the first step gets interpreted as biological variation in the second step. The 254 amount of statistical variation that may be interpreted as biological variation in the linear 255 approximation term to a family of reaction norms is thus $\frac{3 \sigma_{r}^{2}}{n r^{2}}$. Since the residual variance is 256 always positive, the two-step assessment of variation in reaction norm parameters will always 257 be upwardly biased. Because the number of environments will typically be modest $(n$ is 258 the number of points in the regression, and this is typically the number of environmental 259 treatments), this effect can be large. This effect of statistical error in step 1 to contribute to 260 the apparent variation in step 2 will occur in both inferences of average reaction norm slope 261 in estimates of $s_{a b}$, and in regression-based approaches.

262 The basic statistical theory that gives sampling errors of regression parameters can give 263 the sampling variance of quadratic terms $b_{2}$ in the notation introduced above. The sampling 264 error of quadratic terms given a centred uniform covariate is

$$
\Sigma\left[b_{2}\right]=\frac{45 \sigma_{r}^{2}}{4 n r^{4}}
$$

265 See the appendix section A.4 for a more detailed derivation. Comparison of the expressions 266 for sampling variances of the mean, linear, and quadratic terms, i.e., of $\Sigma[\bar{z}], \Sigma\left[b_{1}\right]$ and $\Sigma\left[b_{2}\right]$, 267 reveals a further complication arising in the two-step procedure for inferring variation in 268 reaction norm parameters. The relative contribution of statistical noise to apparent variation 269 in means and linear and quadratic terms (and higher terms pertaining to other aspects of 270 reaction norm curvature) varies depending on the arbitrary scaling of the covariate: the three 271 expressions for sampling variance are different functions of the parameter $r$, the essentially 272 arbitrary range of the environmental variable.

273 The two important points, (1) that statistical noise will be interpreted as biological vari- 
274 ation in two-step analytical procedures, and (2) that the extent to which statistical noise 275 pollutes biological inferences depends on scaling, are not artefacts of the simplifying assump276 tions made here. For example, it occurs if the covariate is not strictly uniform, but rather is 277 composed of few or many distinct environmental treatments. The pattern will also hold for 278 very different distributions of the covariate; analogous expressions for $\Sigma[\mu], \Sigma\left[b_{1}\right]$, and $\Sigma\left[b_{2}\right]$, 279 given a normal covariate, $x$, are given in appendix section A.5.

280 A class of linear mixed models called random regression models exists specifically to sep281 arate noise from real variation in families of regression coefficients. The simplest random 282 regression mixed model is a linear random slopes model, which can be written as

$$
z_{i j}=a+b x_{i}+f_{j}+g_{j} x_{i}+e_{i}
$$

283 where $z_{i j}$ is the phenotypic observation of individual $i$ from group (e.g., species, family, etc.) $284 j$, where $a$ and $b$ are fixed regression parameters for the intercept and slopes, respectively, 285 where $f_{j}$ and $g_{j}$ are regression parameters (contrasts to $a$ and $b$ ) for group $j, x_{i}$ is the 286 environment to which individual $i$ was exposed and $e_{i}$ is a residual for individual $i$. As 287 before, the residuals are assumed to be drawn from a normal distribution $e_{i} \sim N\left(0, \sigma_{r}^{2}\right)$, and 288 furthermore, the group-specific regression parameters are also treated as random variables, 289 i.e., variables that belong to a bivariate normal distribution

$$
\left[\begin{array}{l}
f \\
g
\end{array}\right]_{j} \sim N\left(\mathbf{0},\left[\begin{array}{cc}
\sigma^{2}(f) & \sigma(f, g) \\
\sigma(f, g) & \sigma^{2}(g)
\end{array}\right]\right)
$$

290 such that $\left[\begin{array}{cc}\sigma^{2}(f) & \sigma(f, g) \\ \sigma(f, g) & \sigma^{2}(g)\end{array}\right]$ is a matrix containing the variances and covariances of slopes 291 and intercepts. Solutions to the mixed model give estimates of terms including the variance 292 in slopes $\left(\sigma^{2}(g)\right)$, that are not inflated by sampling error, as occurs in the two-step approach. 293 While random regression analysis is currently in use (Dingemanse et al., 2010; Martin et al., 294 2011), we hope that it is useful to clarify that its use represents more than a mere modernisa- 
295 tion of statistical approaches to studying reaction norms. Random regression can yield direct

296 inferences of variation in reaction norm parameters (e.g., of slopes), that would otherwise be 297 subjected to potentially biologically misleading statistical biases in two-step analyses.

298 A random regression model can be used to assess variation in reaction norms for any 299 analysis with multiple units of observation, and two or more (or a continuous range of) 300 environmental treatments. When applied to a study with two treatments, the linear random 301 regression mixed model specified by equation 5 yields unbiased estimates of the among-group 302 variance in intercepts, average slopes (i.e., this amounts to an analysis of variation in $s_{a b}$ ), 303 and their covariance.

304 When applied to a study with a range of environmental conditions, or with random 305 quadratic (or even higher order) terms, random regression mixed model analysis can be 306 used to recover meaningful information about variation in reaction norm shape. However the 307 caveats that apply to the interpretation of polynomial approximations to reaction norms in 308 general will also apply to inferences about variation in polynomial coefficients obtained by 309 random regression. With prudence, it is possible that random polynomial regression mixed 310 model analysis could be much more extensively used in analysis of variation in reaction 311 norms, and such analysis will certainly be preferable to two-step analytical approaches in 312 most circumstances.

\section{Example application of a random regression mixed model}

314 We applied quadratic random regression mixed model analysis to the data on reaction norms 315 reported in Rocha et al. (2009) and re-analysed in Rocha and Klaczko (2012) (data provided 316 by F. B. Rocha and L. B. Klaczko). The data consist of 1122 Drosophila mediopunctata 317 phenotyped for abdominal spot number and thorax length, raised in three simultaneous 318 replicates (vials) in a thermal gradient spanning $14^{\circ} \mathrm{C}$ to $24^{\circ} \mathrm{C}$ in $1^{\circ} \mathrm{C}$ intervals. For each 
trait, the mixed model took the form

$$
z_{i, j}=a+b_{1} t_{i}+b_{2} t_{i}^{2}+\operatorname{sex}_{i}+f_{j}+g_{1, j} t_{i}+g_{2, j} t_{i}^{2}+\text { replicate }_{i}+T_{i}+e_{i}
$$

320 where $z_{i, j}$ represents the phenotype (spots or thorax length) measured on individual $i$ belong321 ing to strain $j$. The fixed effects, $a, b_{1}$, and $b_{2}$ estimate the average reaction norm, conditional 322 on a fixed effect of sex. The random polynomial coefficients $f_{j}, g_{1, j}$, and $g_{2, j}$ for each line 323 and are assumed to be drawn from a multivariate normal distribution

$$
\left[\begin{array}{l}
f \\
g_{1} \\
g_{2}
\end{array}\right]_{j} \sim N(\mathbf{0}, \mathbf{\Sigma}), \quad \boldsymbol{\Sigma}=\left[\begin{array}{ccc}
\sigma^{2}(f) & \sigma\left(f, g_{1}\right) & \sigma\left(f, g_{2}\right) \\
\sigma\left(f, g_{1}\right) & \sigma^{2}\left(g_{1}\right) & \sigma\left(g_{1}, g_{2}\right) \\
\sigma\left(f, g_{2}\right) & \sigma\left(g_{1}, g_{2}\right) & \sigma^{2}\left(g_{2}\right)
\end{array}\right]
$$

324 with estimated covariance matrix $\boldsymbol{\Sigma}$. Additionally, the replicate associated with individual $i$, 325 and the temperature in which it was raised, coded as a multi-level factor $t_{i}$ and the residuals, $326 e_{i}$, are all included as random effects with estimated variances. The temperature at which a 327 given individual was raised, $t_{i}$ was mean-centred by subtracting $19^{\circ} \mathrm{C}$.

328 The among-line covariance matrices of intercepts, slopes, and curvatures (table 1) are 329 difficult to interpret directly. However, some features of the mixed model analysis are imme330 diately apparent. First, we can see that, as predicted by the statistical theory given above, 331 the variance of coefficients of reaction norms (table 1d) in the two-step procedure inflates 332 the apparent amount of variation in reaction norm parameters. Another such comparison 333 yielding similar inflation of apparent variation in reaction norm parameters is reported in 334 Liefting et al. (2015). This effect is larger in cases when reaction norms are more similar, in 335 this case, with a much more dramatic effect for thorax length reaction norms than for spot 336 number. Furthermore, the correlations among reaction norm parameters are consistently 337 smaller in inferences from the two-step procedure. This is because statistical noise inflates 338 all of the estimates of variance in the polynomial coefficients, but not necessarily all of the 339 covariances. 
However, biological inference based on the estimated variances and covariances of polynomial reaction norm coefficients is difficult. This is because the relationship between reaction

342 norm shape and slopes and intercepts depends on the scaling of the environmental covariate 343 (temperature, in this case). A first step to interpreting the mixed model results might be to 344 visualise the family of reaction norms implied by the fitted mixed model. Figure 2 shows the 345 raw means for each line in each environment (a and b), quadratic regressions fitted for each 346 line (c and d), and an example of 20 reaction norms simulated from the values of the fitted 347 mixed models (e and f). The last depictions are essentially simulations from the inferred 348 distribution of reaction norms, generated by drawing intercepts, linear and quadratic terms 349 from a multivariate normal distribution with a mean defined by the fixed effects in the fitted 350 model, and with (co)variances set to those estimated by the random effects (table 2).

351 While these reaction norms were previously interpreted as showing ubiquitous effects of 352 variation in reaction norm shape, this interpretation seems tenuous based on consideration of 353 the visualisations of the families of reaction norms in figure 2. For spot number, all inferences, 354 including those that inflate the amount of variation in reaction norm shape (parts a and c), 355 indicate that the reaction norms are approximately linear and thus there is in fact only very 356 modest variation in reaction norm shape. A mixed model analysis is particularly useful 357 for separating shared features of reaction norms (characterised by fixed effects) from ways 358 that they vary (characterised by random effects). Indeed, the previous interpretation that 359 these reaction norms show that curvature is a common feature of reaction norms (Rocha 360 and Klaczko, 2012) is true. However, for thorax length, a critical further finding is that 361 the reaction norms of different lines have very similar curvatures; this allows more nuanced 362 interpretation of when and how curvature is an important feature of reaction norms.

363 For thorax length, variation in the reaction norms is even more modest. The crossing of 364 reaction norms has been suggested as a measure of variation in slope, and multiple crossing of 365 reaction norms as a measure of variation in curvature (Rocha and Klaczko, 2012). However, if 366 reaction norms vary very little in any way, then a great deal of crossing occurs! Consider figure 
$3672 \mathrm{~b}$; if all of the reaction norms were nearly identical, then statistical noise in estimating the 368 mean phenotype for each strain in each environment would cause half of the line segments in 369 a plot such as this to cross. Clearly, line-crossing is difficult to apply as a measure of reaction 370 norm complexity.

\section{Variance in phenotype arising from variation in reaction norm parameters}

372 How can we make inferences about the relative importances of variation in the mean values

373 of reaction norms, and of slopes and quadratic terms, if the variances of intercepts, slopes, 374 and quadratic terms depend on the arbitrary scaling and distribution of the environmental 375 covariate (and additionally on the covariance of intercepts and quadratic terms)? Given any 376 distribution of the environmental covariate, it is possible to derive the amount of variation 377 among genetic units (e.g., lines), integrated over the distribution of the covariate, that arises 378 from differences in the mean, slope and quadratic curvature of reaction norms. Detailed 379 derivations are given in the appendix (section A.6 for a uniform covariate and A.7 for a 380 normal covariate), and the formulae for these measures of variation in different aspects of 381 reaction norm shape are given in table 2.

382 Figure 3 shows the amount of variation in expected line- and temperature-specific pheno383 typic values for both traits in the Drosophila example that are attributable to variation in the 384 means, slopes, and curvatures of the families of reaction norms, as assessed by the quadratic 385 random regression mixed model analysis (equation 6), and by the two-step analytical pro386 cedure. This quantification of different components of variation in reaction norms confirms 387 that differences in reaction norm shape are indeed modest, and also further demonstrates 388 the danger of inflating inferences in the two-step analytical procedure (figure 3a,b). The 389 majority of variation among lines arises from differences in mean values of reaction norms.

390 Calculations of variation attributable to intercepts, linear, and quadratic terms, separately 391 made based on uniform and normal distributions, are quite similar in this example (compare 392 figures 3a,b with 3c,d). This should generally be the case when families of quadratic functions 
393 capture variation in reaction norms well, as seems to be the case for these data.

394 (Co)variances of arbitrary reaction norm properties

395 Intercepts, linear, and quadratic terms, and the variance in expected values with which they 396 are associated, do not directly represent all features of reaction norms in which we may be 397 interested. For example, we may be interested in phenotypic values at specific environmen398 tal values, mean phenotypic values integrated over different distributions of environmental 399 values, locations of maxima or minima (environments that produce minimum or maximum 400 phenotypes), and phenotypic values at maxima or minima (minimum or maximum phenotypic 401 values). Quadratic regressions contain information about such reaction norm properties, pre402 viously sometimes termed "characteristic values" (Delpuech et al., 1995; Gibert et al., 1998), 403 and families of regression coefficients, as estimable by random regression analysis, contain 404 information about means and variances of such reaction norm properties. Operationally, 405 calculations of variance in arbitrary reaction norm properties seems easiest in the two-step 406 analytical procedure. In mixed model analyses, one must call on somewhat more statisti407 cal sophistication to derive (co)variances of reaction norm properties from estimated means, 408 variances, and covariances of quadratic reaction norm parameters. However, an approach 409 to develop formulae for such quantities seems clear. Given a function for calculating some 410 quantity (e.g., the location of an optimum) from a fitted reaction norm function, the vari411 ance of that quantity can be approximated by taking the expectation of a Taylor series. It 412 is reasonable to feel that this is easier said than done. It may therefore be useful to provide 413 expressions involving some quantities that might be most useful.

414 Table 3 gives expressions for quantities that may be calculated from quadratic reaction 415 norm approximations: the environment of the maximum or minimum value, the maximum or 416 minimum value, and the mean (remembering that the intercept is not the mean of a quadratic 417 function, even if the covariate is mean centred) for different distributions of the environmental 418 covariate. Table 3 also gives expressions for the expectations and variances of each of these 
419 quantities, given means and variances and covariances of quadratic regression coefficients, as 420 are obtained from random regression mixed model analysis. Similar approaches to those that 421 yielded these expressions (given in appendix section A.8), can be used to give variances and 422 covariances of multiple derived reaction norm properties.

423 We can briefly explore the application methods to infer distributions of arbitrary reaction 424 norm properties using the data on the reaction norms of thorax length as a function of 425 temperature (table 1, figure 2). While mean values for spot number may be biologically 426 informative, means and variances of locations and values of optima for spot number will 427 not. This is because the distribution of reaction norms contains very many nearly linear 428 functions (figure 2), and optima of the quadratic approximations of such functions are far 429 from the relevant range of temperature. However, the distribution of reaction norms for 430 thorax length appears to have a reasonably well-defined maximum, and knowing how this 431 maximum's value and location varies among reaction norms may be of biological interest. 432 The mean and variance of locations of maxima for thorax length, as given by the expressions 433 in table 3 are 15.7 and 1.11, respectively. Means and variances of the phenotypic values at 434 the maxima are 1.56 and $2.93^{-4}$, respectively.

435 The approach to obtaining expressions for (approximating) the distributions of arbitrary 436 reaction norm properties, as given in table 3 and appendix section A.8, could be extended in 437 order to obtain other metrics of potential interest, for example, the covariance of locations 438 and values of optima; however, the expressions will become increasingly unwieldy. The main 439 value of the expressions given in table 3 is that they demonstrate that the random regres440 sion approach provides the information necessary to infer arbitrary properties of families of 441 reaction norms, given the assumption that a family of quadratic functions gives a reasonable 442 approximating model. A more pragmatic option is available. Monte-Carlo (MC) simulation 443 can provide very precise approximations to quantities such as means and variances of arbi444 trary quantities. In the present setting, the technique would require simulation of a large 445 number (say a million) random normal vectors with means and covariances equal to those 
446 estimated by the fixed and random parts of a quadratic random regression mixed model. 447 Then for each simulated vector, one or more quantities of interest can be calculated. The 448 means, variances, and covariances of these simulated reaction norm properties will approach 449 the values defined by the fitted random regression mixed model, as the number of simulations 450 becomes large. On modern personal computers, this kind of procedure takes seconds. By 451 this procedure (with one million MC simulations) the mean of the environments of maximum 452 values, and the mean of the maxima, are 15.6 and 1.56. The variance of environments of 453 maximum values is 1.29 , the variance of maximum values is $3.18^{-4}$, and the covariance of the 454 two quantities is $-1.01^{-2}$ (the associated correlation is -0.497). The first four of these statistics 455 agree very closely with the four values given above based on approximations given in table 3 , 456 indicating that the approximations may generally be robust. Further, it may be of particular 457 interest in a biological example such as this, that those lines with the highest optima have 458 their optima at the lowest temperatures. MC simulation approaches can be applied as well 459 to obtaining standard errors of statistics of the distributions of arbitrary quantities.

460 Another property of sets of reaction norms that may be of biological interest is the 461 environment-specific variance of traits, and covariance among genetic units across environ462 ments. Any parameters describing the variances and covariance of reaction norm parameters 463 (e.g., intercepts, slopes, etc.) defines a specific pattern of within- and across-environmental 464 (co)variance in phenotype. We have focused so far on a reaction norm, or "function-valued 465 trait" approach to phenotypic plasticity, but this perspective is entirely complimentary to 466 thinking about environment-specific covariances, which is sometimes called the "character 467 state" approach (van Tienderen and Koelewijn, 1994). For example, in Box 1 figure B1a, 468 a we could make a character state description of the reaction norms by saying that there is 469 little variance in the trait associated with genotypes at $x=4$, but appreciable variance for 470 higher an lower values of the environmental variable; furthermore, we could state that, at 471 the genetic level, trait values when $x<4$ are negatively correlated with trait values when $472 x>4$. A character state representation can sometimes be a very useful way of describing the 473 properties of a family of covarying reaction norm parameters. Box 2 provides a description 
474 of how to represent covariances of reaction norm parameters as environment-specific means 475 and covariances.

476 The quantities discussed in this and the previous section for summarising reaction norms 477 apply directly to families of quadratic regressions (and associated formulae in tables 2 and 478 3), especially as can be estimated with random regression approaches in linear mixed models. 479 The reasoning behind these could in principle be extended to other types of functions, for 480 example, to higher-order polynomials. In analyses of non-normal traits, e.g., with random 481 regression in generalised mixed models, quantities described here would apply on the under482 lying latent scale (e.g., on the log scale in a Poisson model), which in many cases could be 483 very useful and biologically interpretable.

\section{Discussion and Conclusions}

485 We are neither advocating for, nor against, characterising reaction norm slope as the slope 486 of a line between two points, i.e., of $s_{a b}$, on the reaction norm. We seek primarily to clarify 487 that this very simple statistic has a very specific interpretation (i.e., the average slope of the 488 reaction norm between points $a$ and $b$ ) that holds regardless of the true shape of the reaction 489 norm. Few other statistics one might use to characterise reaction norms have interpretations 490 that holds so generally. This does not mean that $s_{a b}$ could not potentially be misleading. For 491 example, if a reaction norm contains a minimum or a maximum between the points $x=a$ 492 and $x=b$, then it is difficult to see what biological use inferences of $s_{a b}$ may be, without fur493 ther detailed analyses of reaction norm shape. Thus, there are situations where $s_{a b}$ contains 494 exactly the information that is needed, even if a reaction norm is highly non-linear, and there 495 are times when information is needed that $s_{a b}$ cannot provide. Understood correctly, there is 496 neither a "pessimistic" nor an "optimistic" view (Rocha and Klaczko, 2012) to be had about $497 s_{a b}$; rather it is fit for some purposes and irrelevant to others. Additionally, it is of note that 498 the distribution of slopes and intercepts of straight-line reaction norms between two environ499 ments has direct relationships to cross-environment phenotypic and genetic correlations (Via 
500 and Lande, 1985), another simple and robust relationship that does not necessarily hold for 501 more complex statistical models of reaction norms.

502 Similarly, while we have attempted to be very careful about the narrowness of the con503 ditions under which coefficients of polynomial regressions can be interpreted as reflecting 504 specific properties of true reaction norms, we are neither advocating for, nor against, poly505 nomial regression. In particular, we note that polynomial approximating functions depend 506 on the distribution of the covariate (i.e., the environmental variable), and cannot therefore 507 necessarily be interpreted solely as properties of reaction norms. More importantly, we have 508 noted that the slope of an approximation to a reaction norm at any specific point (i.e., Rocha 509 and Klaczko 2012's "local plasticity") is not necessarily a good representation of the slope 510 of the true reaction norm at that point. If a polynomial regression is sufficiently flexible 511 relative to the presumed complexity of a true reaction norm, then this technique is likely to 512 be valuable, even if it does not generally yield estimates of quantities that have such a simple 513 and general interpretation as estimates of $s_{a b}$. We suspect that quadratic regression could 514 prove to be a very pragmatic model of many non-linear reaction norms.

515 Despite the previous claim that the Drosophila data in figure 2 support a contention that 516 reaction norms vary extensively in their curvature, it is fairly easy to see that this is not 517 the case. Taking into consideration that finite sample size for each line in each assayed 518 temperature causes statistical noise in each point in figure $2 \mathrm{a}, \mathrm{b}$, it is clear that there is very 519 little variation in reaction norm shape among lines. For abdominal spot number, the raw 520 data consist primarily of parallel lines. For thorax length, dispassionate visual inspection and 521 quantitative analysis shows that most of the variation is explained by the overall reaction 522 norm, and by variation within temperatures, not differences in reaction norms among lines. 523 Note that quantitative approaches (table 1, figure 5) support these contentions based on 524 the raw data. Since two-step analytical procedures have been widely used in the primary 525 literature, and even meta-analysis (Murren et al., 2014), it is not currently possible to judge 526 how flexible polynomial regressions might generally have to be to capture the most important 
527 features of (variation in) reaction norms. However, the analyses here are heartening and it 528 seems plausible, that with due consideration to the features of any particular study system, 529 that quadratic regressions, as advocated by (Rocha and Klaczko, 2014), could indeed provide 530 pragmatic models of reaction norms in many cases.

531 However, we caution strongly against some of Rocha and Klazcko's (2014) specific sugges532 tions for interpreting quadratic regressions. In particular, Rocha and Klazcko suggest that 533 the derivative of a quadratic, or other polynomial function at any specific point, which they 534 call "local reaction norm plasticity" could be a generally useful measure of reaction norm 535 shape at a particular point. However, this derivative need not necessarily closely reflect the 536 slope of a true reaction norm at that point, and it need not even be the correct sign (see also 537 appendix section 2, figures A.1 and A.2). Rocha and Klaczko (2014) also suggest that the 538 quadratic term can be used as a measure of "reaction norm shape", justified by the fact that 539 twice the value of the quadratic term is the second derivative of the quadratic function at 540 all points, and is therefore the average derivative of the quadratic function. This use seems 541 reasonable, but its application should be approached with awareness that the average deriva542 tive or second derivative of the quadratic approximation to any function is only equal to the 543 average derivative or second derivative under two conditions. First, this equality holds if the 544 true function is indeed quadratic. Second, this equality holds if the environmental covariate 545 is normal. Virtually no studies of reaction norms have a normal covariate. In fact, investi546 gators typically strive for covariate distributions that approach uniformity. So justification 547 for using curvature of a quadratic approximation as a measure of the curvature of a reaction 548 norm rests on a requirement that a quadratic function is a good approximation of the true 549 reaction norm. It seems that this requirement should frequently be closely enough met in 550 empirical systems for quadratic regression to provide useful measures of average reaction 551 norm curvature.

552 We do advocate more strongly for analyses that do not apply statistical procedures to 553 the outcomes of previous statistical procedures. Doing statistics on statistics will often lead 
554 to misleading results, and is generally avoidable. Although some authors have begun to 555 use mixed model analysis (e.g., Dingemanse et al. 2010; Martin et al. 2011), multi-step ap556 proaches are still common empirical practice. Furthermore, while the applicability of random 557 regression has been clearly demonstrated (Martin et al., 2011), we hope it is useful to clarify 558 that it doesn't merely represent a modernisation of statistical approaches to studying reaction 559 norms. Rather, it allows pitfalls of two-step procedures to be avoided. When population-level 560 variation is inferred from the distribution of summary statistics of units of organisation that 561 have been analysed within that population, the apparent variation at the population-level 562 will invariably be upwardly biased by statistical noise (see also Morrissey 2016). Given the 563 existence of random regression mixed models in widely-used software packages (e.g., LME4, 564 Bates et al. 2014; MCMCGLmm, Hadfield 2010; ASRemL, Gilmour et al. 2002) their use in 565 studies of reaction norms should probably be extended. However, just as the biological utility 566 of polynomial approximations to reaction norm functions depends on the closeness to which 567 they approximate true reaction norm functions, inferences from random regression mixed 568 models will also depend on the adequacy of a family of polynomial functions to describe 569 variation in reaction norms.

570 Additional benefits of mixed model-based analyses that we realised in our example anal571 ysis also contribute to the utility of these methods. For example, we were able to account 572 for covariates (by fitting a fixed effect for sex), and possible sources of variation and non573 independence among observations (by treating variation among replicate vials as random 574 effects). Furthermore, mixed-model analyses can very naturally account for unequal num575 bers of observations in different environmental treatments, while such accounting is more 576 difficult in the two-step procedure (weighting by precision would be necessary, and it is not 577 clear if such an effort has ever been made in reaction norm studies). These seem like valuable 578 aspects of the analysis for ensuring the most complete and rigorous use of the available data, 579 and are relatively easily implemented in the mixed model framework. Furthermore, when 580 a low-order polynomial function does not fit a reaction norm well, mixed model analysis 581 may provide simple and powerful solutions. For example, the average reaction norm may be 
582 handled with the fixed effects part of a model, using a high-order polynomial or some other 583 flexible regression function such as a spline regression (Wood, 2006), while a relatively low584 order model, such as a quadratic random regression, may still be pragmatic for describing 585 variation in reaction norms around the average function.

586 Linear mixed models, in particular the random regression mixed models considered here, 587 are among the simplest of types of hierarchical model that may be useful to analysis of 588 reaction norm shapes. Non-linear mixed models, and hierarchical models in general, could 589 potentially be used to provide direct inference of variation in parameters such as the locations 590 of maxima, and for coefficients of reaction norm models that are not based on polynomials. 591 Flexible models that can provide such inferences are becoming increasingly easy to implement, 592 for example with software such as JAGS (Plummer, 2010) and STAN (Stan Development Team, 593 2014). With this range of options for model-based inference of variation in reaction norms, it 594 should be increasingly possible to design powerful studies of interesting aspects of phenotypic 595 plasticity.

\section{Acknowledgements}

597 F. B. Rocha and L. B. Klaczko provided the Drosophila data. Mike Ritchie and Kerry John598 son provided comments on earlier drafts of this paper, which was also greatly improved by 599 comments from two anonymous reviewers. MBM is supported by a Royal Society (Lon600 don) University Research Fellowship. ML is supported by the Netherlands Organisation for 601 Scientific Research, VIDI grant nr. 864.03.003.

\section{References}

603 Bates, D., M. Maechler, B. Bolker, and S. Walker, 2014. lme4: Linear mixed-effects models 604 using Eigen and S4, R package version 1.1-7 ed. 
605 Berg, M. P., E. T. Kiers, G. Driessen, M. van der Heijden, B. W. Kooi, F. Kuenen, M. Liefting, 606 H. A. Verhoef, and J. Ellers. 2010. Adapt or disperse: understanding species persistence 607 in a changing world. Global Change Biology 16:587-598.

608 Chevin, L. M., R. Lande, and G. M. Mace. 2010. Adaptation, plasticity, and extinction in a 609 changing environment: towards a predictive theory. PLOS Biology 8:e1000357.

610 Delpuech, J.-. M., B. Moreteau, J. Chiche, E. Pla, J. Vouidibio, and J. R. David. 1995. Phe611 notypic plasticity and reaction norms in temperate and tropical populations of Drosophila 612 melanogaster: ovarian size and developmental temperature. Evolution 49:670-675.

613 DeWitt, T. J. and S. M. Scheiner, 2004. Phenotypic plasticity - Functional and Conceptual 614 Approaches. Oxford University Press, Oxford.

615 Dingemanse, N. J., A. J. Kazeem, K. Réale, and J. Wright. 2010. Behavioural reaction 616 norms: animal personality meets individual plasticity. Trends in Ecology and Evolution $617 \quad 25: 81-89$.

618 Ellers, J. and G. Driessen. 2011. Genetic correlation between temperature-induced plasticity 619 of life-history traits in a soil arthropod. Evolutionary Ecology 25:473-484.

620 Fallis, L., J. Fanara, and T. Morgan. 2014. Developmental thermal plasticity among 621 Drosophila melanogaster populations. Journal of Evolutionary Biology 27:557-564.

622 Gavrilets, S. and S. M. Scheiner. 1993. The genetics of phenotypic plasticity. V. Evolution 623 of reaction norm shape. Journal of Evolutionary Biology 6:31-48.

624 Geyer, C. J. and R. G. Shaw, 2010. Aster models and the Lande-Arnold beta. Tech. rep., 625 University of Minnesota.

626 Ghalambor, C. K., J. K. McKay, S. P. Carroll, and D. N. Reznick. 2007. Adaptive versus 627 non-adaptive phenotypic plasticity and the potential for contemporary adaptation in new 628 environments. Functional Ecology 21:394-407. 
629 Gibert, P., B. Moreteau, J. R. David, and S. M. Scheiner. 1998. Describing the evolution of

630 reaction norm shape: body pigmentation in Drosophila. Evolution 52:1501-1506.

631 Gilmour, A. R., B. J. Gogel, B. R. Cullis, S. J. Welham, and R. Thompson, 2002. ASReml 632 user guide release 1.0. VSN International Ltd, Hemel Hempstead, United Kingdom.

633 Gupta, A. P. and R. C. Lewontin. 1982. A study of reaction norms in natural populations 634 of Drosophila pseudoobscura. Evolution 36:934-948.

635 Hadfield, J. 2010. MCMC methods for multi-response generalized linear mixed models: The 636 MCMCglmm R package. Journal of Statistical Software 33:1-22.

637 Kingsolver, J. G., R. Izem, and G. J. Ragland. 2004. Plasticity of size and growth in fluctuat638 ing thermal environments: comparing reaction norms and performance curves. Integrative 639 and Comparative Biology 44:450-460.

640 Kirkpatrick, M., D. Lofsvold, and M. Bulmer. 1990. Analysis of the inheritance, selection, 641 and evolution of growth trajectories. Genetics 124:979-993.

642 Lande, R. and S. J. Arnold. 1983. The measurement of selection on correlated characters. 643 Evolution 37:1210-1226.

644 Liefting, M., R. H. A. van Grunsven, M. B. Morrissey, M. J. T. N. Timmermans, and 645 J. Ellers. 2015. Interplay of robustness and plasticity of life-history traits drives ecotypic 646 differentiation in thermally distinct habitats. Journal of Evolutionary Biology 28:1057$647 \quad 1066$.

648 Liefting, M., A. A. Hoffmann, and J. Ellers. 2009. Plasticity versus environmental canaliza649 tion: population differences in thermal responses along a latitudinal gradient in Drosophila 650 serrata. Evolution 63:1954-1963.

651 Martin, J. G. A., D. H. Nussey, A. J. Wilson, and D. Réale. 2011. Measuring individual 652 differences in reaction norms in field and experimental studies: a power analysis of random 653 regression models. Methods in Ecology and Evolution 2:362-374. 
654 Mitchell-Olds, T. and R. G. Shaw. 1987. Regression analysis of natural selection: statistical 655 inference and biological interpretation. Evolution 41:1149-1161.

656 Morin, J. P., B. Moreteau, G. Pétavy, and J. R. David. 1999. Divergence of reaction norms 657 of size characters between tropical and temperate populations of Drosophila melanogaster 658 and D. simulans. Journal of Evolutionary Biology 12:329-339.

659 Morrissey, M. B. 2016. Meta-analysis of magnitudes, differences, and variation in evolutionary 660 parameters. Journal of Evolutionary Biology in press.

661 Murren, C. J., H. J. Maclean, S. E. Diamond, U. K. Steiner, M. A. Heskel, C. A. Handels662 man, C. K. Ghalambor, J. R. Auld, H. S. Callahan, D. W. Pfennig, R. A. Relyea, C. D. 663 Schlichting, and J. G. Kingsolver. 2014. Evolutionary change in continuous reaction norms. 664 The American Naturalist 183:453-467.

665 Nussey, D. H., E. Postma, P. Gienapp, and M. E. Visser. 2005. Selection on heritable 666 phenotypic plasticity in a wild bird population. Science 310:304-306.

667 Pétavy, G., J. R. David, P. Gilbert, and B. Moreteau. 2001. Viability and rate of development 668 at different temperatures in Drosophila: A comparison of constant and alternating thermal 669 regimes. Journal of Thermal Biology 26:29-39.

670 Plummer, M., 2010. JAGS version 2.0 Manual. International Agency for Research on Cancer.

671 Rocha, F. B. and L. B. Klaczko. 2012. Connecting the dots of nonlinear reaction norms 672 unravels the threads of genotype-environment interaction in Drosophila. Evolution 66:34046733416.

674 -2014. Undesirable consequences of neglecting nonlinearity: response to comments 675 by Liefting et al. (2013) on Rocha and Klaczko (2012). Evolution 68:1548-1551.

676 Rocha, R. B., H. F. Medeiros, and L. B. Klaczko. 2009. The reaction norm for abdominal 677 pigmentation and its curve in Drosophila mediopunctata depend on the mean phenotypic 678 value. Evolution 63:280-287. 
679 Scheiner, S. M. 1993. Genetics and evolution of phenotypic plasticity. Annual Review of 680 Ecology, Evolution and Systematics 24:35-68.

681 Scheiner, S. M. and H. S. Callahan. 1999. Measuring natural selection on phenotypic plas682 ticity. Evolution 53:1704-1713.

683 Schmalhausen, I. I., 1949. Factors of Evolution. Blakinston, Philedelphia, PA.

684 Stan Development Team, 2014. Stan: A C++ Library for Probability and Sampling, Version $685 \quad 2.5 .0$.

686 Stein, C. M. 1973. Estimation of the mean of a multivariate normal distribution. Proceedings 687 of the Prague Symposium on Asymptotic Statistics 1:345-381.

688 van Tienderen, P. H. and H. P. Koelewijn. 1994. Selection on reaction norms, genetic 689 correlations, and constraints. Genetical Research 64:115-125.

690 Via, S. and R. Lande. 1985. Genotype-environment interaction and the evolution of pheno691 typic plasticity. Evolution 39:505-522.

692 West-Eberhard, M. J., 2003. Developmental plasticity and evolution. Oxford University 693 Press, Oxford.

694 Woltereck, R. 1909. Weitere experimentelle Untersuchungen über Artveränderung, speziel 695 uber das Wesen quantitativer Artunterschiede bei Daphniden. Verhandlungen der 696 Deutschen Zoologischen Gesellschaft 19:110-173.

697 Wood, S. N., 2006. Generalized Additive Models: An Introduction with R. Chapman and 698 Hall/CRC. 
Table 1: Random regression mixed model-based inference of variation in reaction norms for spot number and thorax length in Drosophila mediopunctata, (a) fixed effect estimates, (b) standard deviations of random intercepts, linear, and quadratic terms, and their correlations, and (c) standard deviations of additional random effects. For comparison, standard deviations and correlations of intercepts, linear, and quadratic terms from a traditional multi-step procedure are given in part (d).

\begin{tabular}{|c|c|c|c|c|c|c|}
\hline \multicolumn{4}{|c|}{ number of spots } & \multicolumn{3}{|c|}{ thorax length } \\
\hline \multicolumn{7}{|c|}{ (a) fixed effects } \\
\hline$a$ & 1.8 & & & 1.54 & & \\
\hline$b_{1}$ & -0.162 & & & $-1.21^{-2}$ & & \\
\hline$b_{2}$ & $-4^{-4}$ & & & $-1.9^{-3}$ & & \\
\hline sex & 0.243 & & & -0.156 & & \\
\hline \multicolumn{7}{|c|}{ (b) random quadratic regression coefficients (as SDs and correlations) } \\
\hline & $f$ & $g_{1}$ & $g_{2}$ & $f$ & $g_{1}$ & $g_{2}$ \\
\hline$f$ & 0.618 & & & $1.54^{-2}$ & & \\
\hline$g_{1}$ & -0.263 & 0.0485 & & 0.117 & $2.3^{-3}$ & \\
\hline$g_{2}$ & -0.979 & 0.454 & $1.35^{-2}$ & -0.243 & -0.992 & $2^{-4}$ \\
\hline \multicolumn{7}{|c|}{ (c) additional random variance components (as SDs) } \\
\hline replicate & 0.158 & & & 0.0212 & & \\
\hline temperature & 0.0382 & & & $6.6^{-3}$ & & \\
\hline residual & 0.477 & & & 0.0571 & & \\
\hline \multicolumn{7}{|c|}{ (d) SDs and correlations of polynomial coefficients from the two-step procedure } \\
\hline & $f$ & $g_{1}$ & $g_{2}$ & $f$ & $g_{1}$ & $g_{2}$ \\
\hline$f$ & 0.642 & & & 0.0299 & & \\
\hline$g_{1}$ & -0.0802 & 0.0447 & & -0.159 & $7^{-3}$ & \\
\hline$g_{2}$ & -0.933 & 0.286 & $1.69^{-2}$ & -0.671 & $-1.25^{-2}$ & $1.2^{-3}$ \\
\hline
\end{tabular}

Table 2: Expressions for variance in environment-specific expected values of phenotype attributable separately to variation in reaction norm means, slopes, and curvatures (quadratic terms), for uniform and normal environmental covariates. All expressions assume that covariates are mean-centred. For the uniform distribution, $r$ represents the range, i.e., where the centred uniform covariate has a range from $-r$ to $+r$. For the normal covariate, the environmental variable's distribution is characterised by the standard deviation, $\sigma$.

\begin{tabular}{lcc}
\hline \hline & uniform & normal \\
\hline mean $\left(\Sigma_{\mu}\right)$ & $\frac{1}{9}\left(6 r^{2} \sigma\left(f, g_{2}\right)+9 \sigma^{2}(f)+r^{4} \sigma^{2}\left(g_{2}\right)\right)$ & $\sigma^{2}(f)+2 \sigma\left(f, g_{2}\right) \sigma^{2}(x)+\sigma^{2}\left(g_{2}\right) \sigma^{4}(x)$ \\
slopes $\left(\Sigma_{g_{1}}\right)$ & $\frac{1}{3} r^{2} \sigma^{2}\left(g_{1}\right)$ & $\sigma^{2}\left(g_{1}\right) \sigma^{2}(x)$ \\
curvatures $\left(\Sigma_{g_{2}}\right)$ & $\frac{4}{45} r^{4} \sigma^{2}\left(g_{2}\right)$ & $2 \sigma^{4}(x) \sigma^{2}\left(g_{2}\right)$ \\
\hline \hline
\end{tabular}




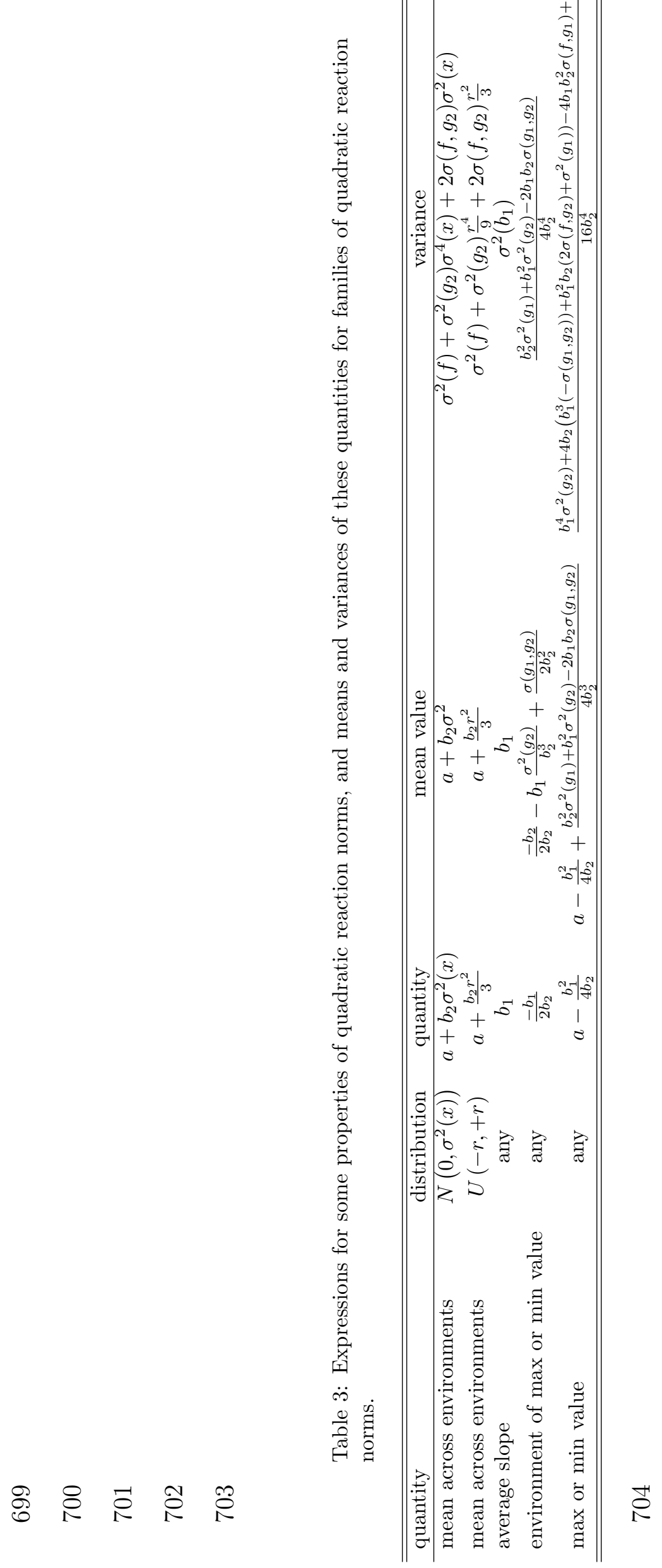



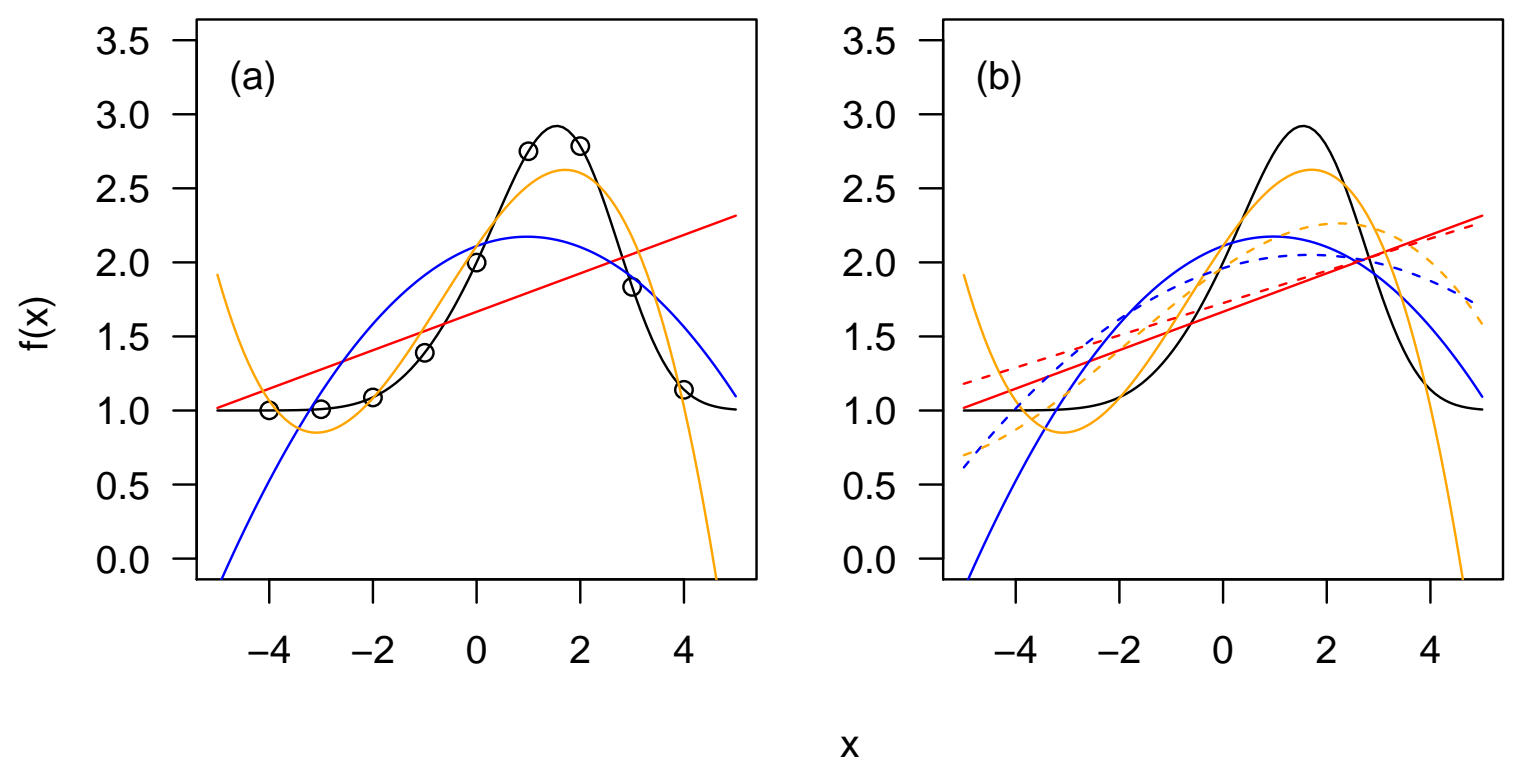

Figure 1: Polynomial approximations to a non-linear reaction norm. The black curve represents an hypothetical true reaction norm of the form $E(z)=1+e^{0.75 x-0.15 x^{2}-0.04|x|^{3}}$, which has the basic shape often expected for a thermal performance reaction norm. The solid lines show the predictions of polynomial approximations of the reaction norm of first- (red), second- (blue) and third-order (orange). Panel (a) shows the polynomial approximations assuming that nine environment-specific population mean phenotypes are known with essentially no error (e.g., as though there were very high sample sizes). Panel (b) repeats the true (black line) and approximated (solid coloured lines) reaction norms from (a), and in addition shows three more approximations of the reaction norm, in dashed lines. These are the polynomial approximations to the reaction norm that would be obtained, given the same true reaction norm, but if the environmental covariate was normally-distributed with mean zero, and with the same variance as among the nine treatments in part (a). 

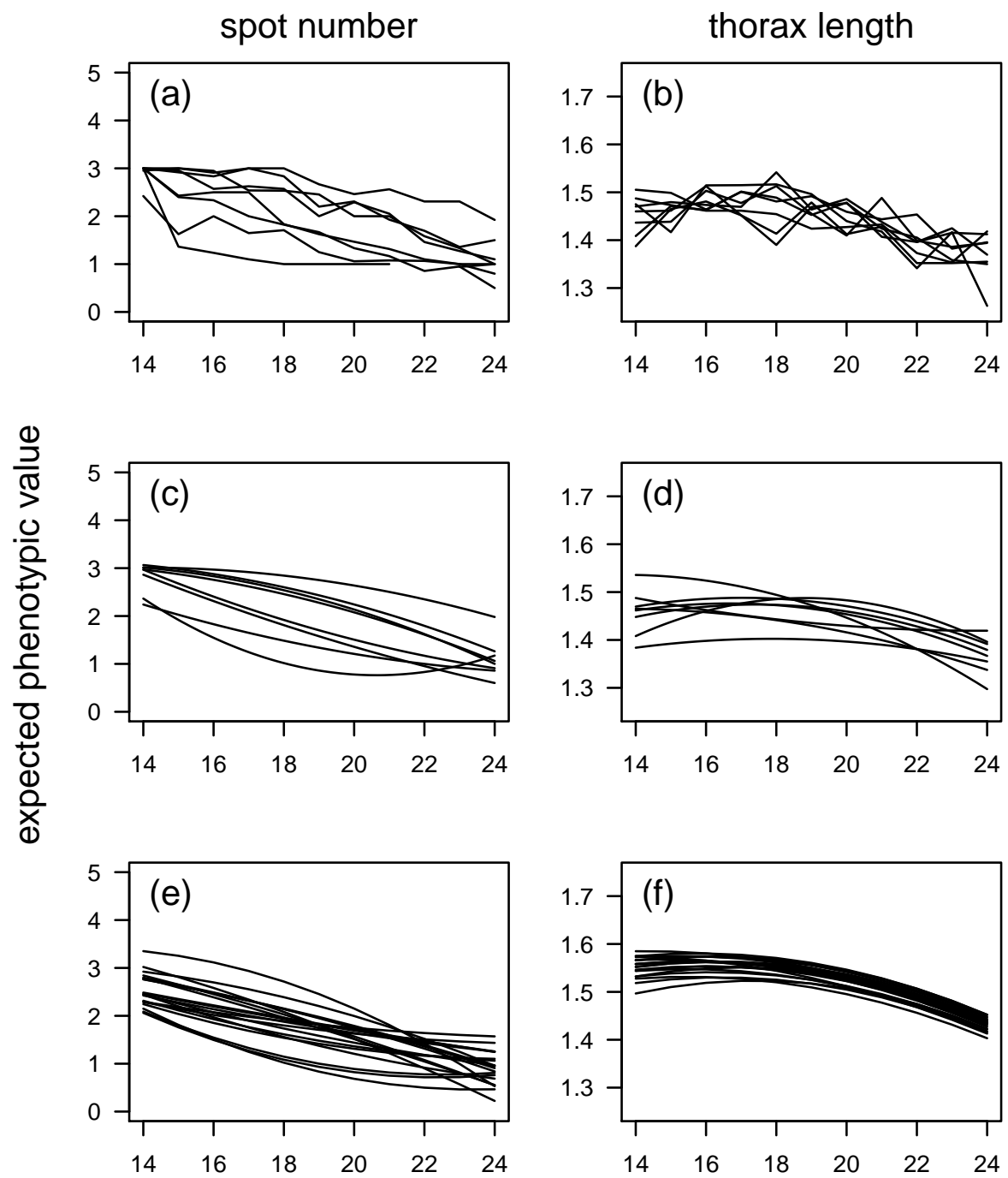

temperature

Figure 2: Representations of variation in reaction norm shape for abdominal spot number (left column) and thorax length (right column) among eight strains of Drosophila mediopunctata (raw data from Rocha et al. 2009). (a) and (b) show strain- and temperature-specific means, (c) and (d) show quadratic approximations to the strain-specific mean temperatures. (e) and (f) show families of 20 simulated reaction norms from quadratic random regression mixed models fitted to the individual-based (rather than line mean) data. 


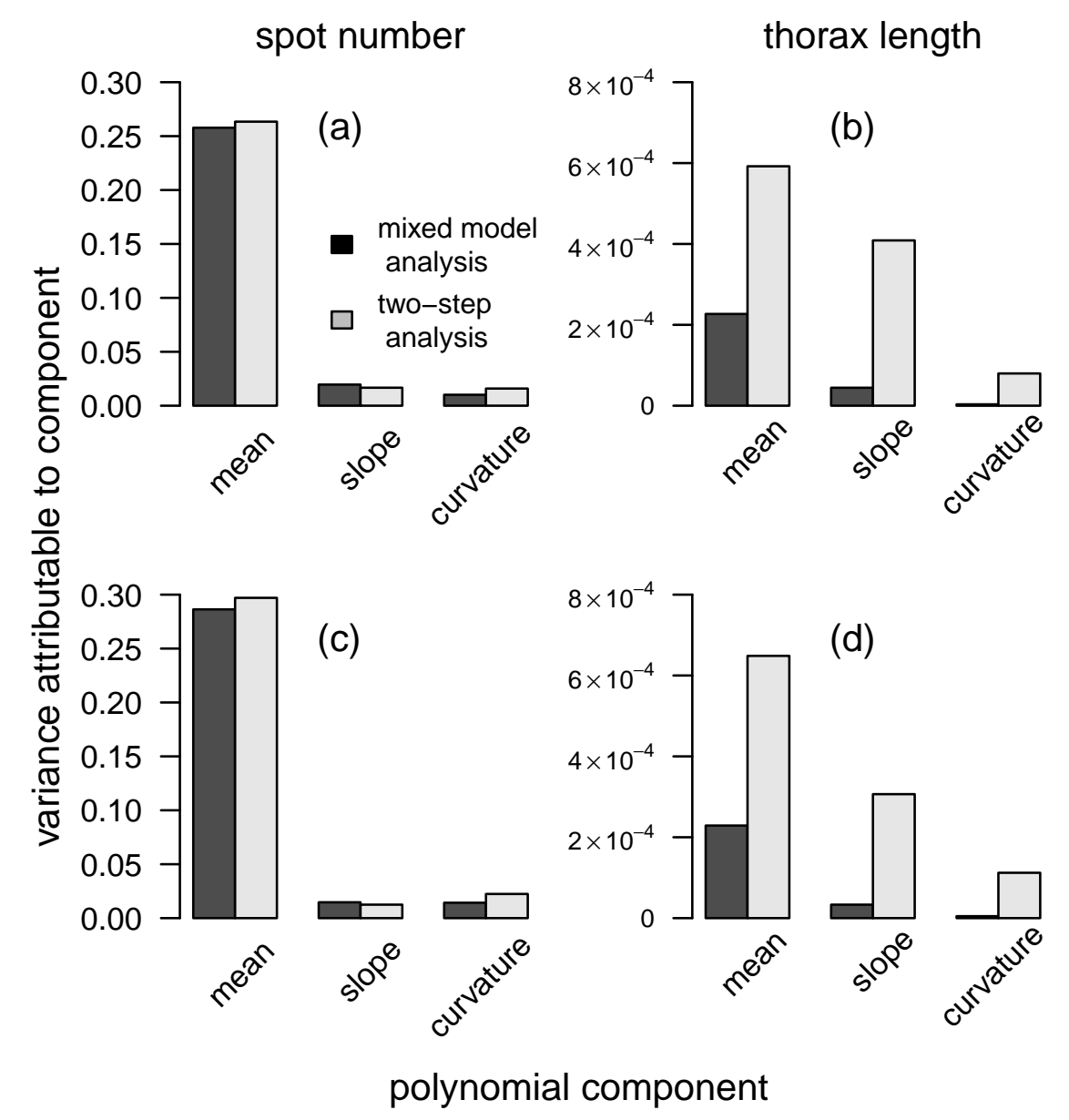

Figure 3: Proportions of variation around the average thermal reaction norms of abdominal spot number and thorax length in strains of Drosophila mediopunctata (raw data from Rocha et al. 2009), attributable to variation in overall means, slopes, and intercepts. (a) and (b) are calculated for a uniform covariate from 14 to 24 degrees (i.e., $r=5$ ). (c) and (d) are calculated for a normal covariate centred on 19 degrees, and with a standard deviation of $r / 2$, such that approximately 95 percent of the values of the environmental covariate would fall in the range investigated (i.e., between 14 and 24). Black bars show variances attributable to the polynomial components derived from a mixed model analysis (as depicted in figure 4e,f), and grey bars show those from the two-step analytical procedure. 


\section{Box 1: Mean centring of covariates}

706 In linear regression analyses of reaction norms, one may be interested in how much mean

707 values vary, and how much slopes vary (and perhaps how means and slopes covary). The

708 intercept only represents the mean if the environmental covariate is mean-centred. Figure

709 B1.1 illustrates why this is so: if functions are sloped, then intercepts can be very different

710 from mean values. If two regression functions have different slopes, then differences in their

711 intercepts may be very different from differences in their mean values, if the covariate is 712 not mean-centred (figure B1.1a). Intercepts do represent the means for a centred covariate, 713 regardless of how slopes differ among genetic units (figure B1.1a). Furthermore, mean714 centring can alleviate artifactual correlations among parameters. While there is no correlation 715 of means and slopes in the reaction norms depicted in figure B1.1 parts a and b - in fact 716 there is no variation in means - slopes and intercepts are highly correlated in part a, but the 717 true biological pattern of no covariance is reflected under mean-centring, as depicted in b.

718 Similarly to how intercepts cannot be interpreted as mean values of a regression function 719 when a covariate is not centred, linear terms may be unrelated to average slopes of quadratic 720 regression functions, unless the covariate is mean-centred (and symmetric). Linear terms in 721 quadratic regression models are the slope of the function at the point where the covariate 722 is zero. Consider figure B1.1c: the two depicted lines have identical average slopes over the 723 range of the environment from three to five, and identical slopes at $x=4$. However, if 724 three to five environmental units is the range over which data have been collected, these two 725 regressions will have opposite linear terms in a regression analysis where the covariate is not 726 centred. Figure B1.1d depicts regression analyses of the same data, but with a mean-centred 727 covariate, where it is evident that the linear terms will have the same value, reflecting the 728 fact that the two approximating functions have the same slope at the mean value of the 729 covariate. 

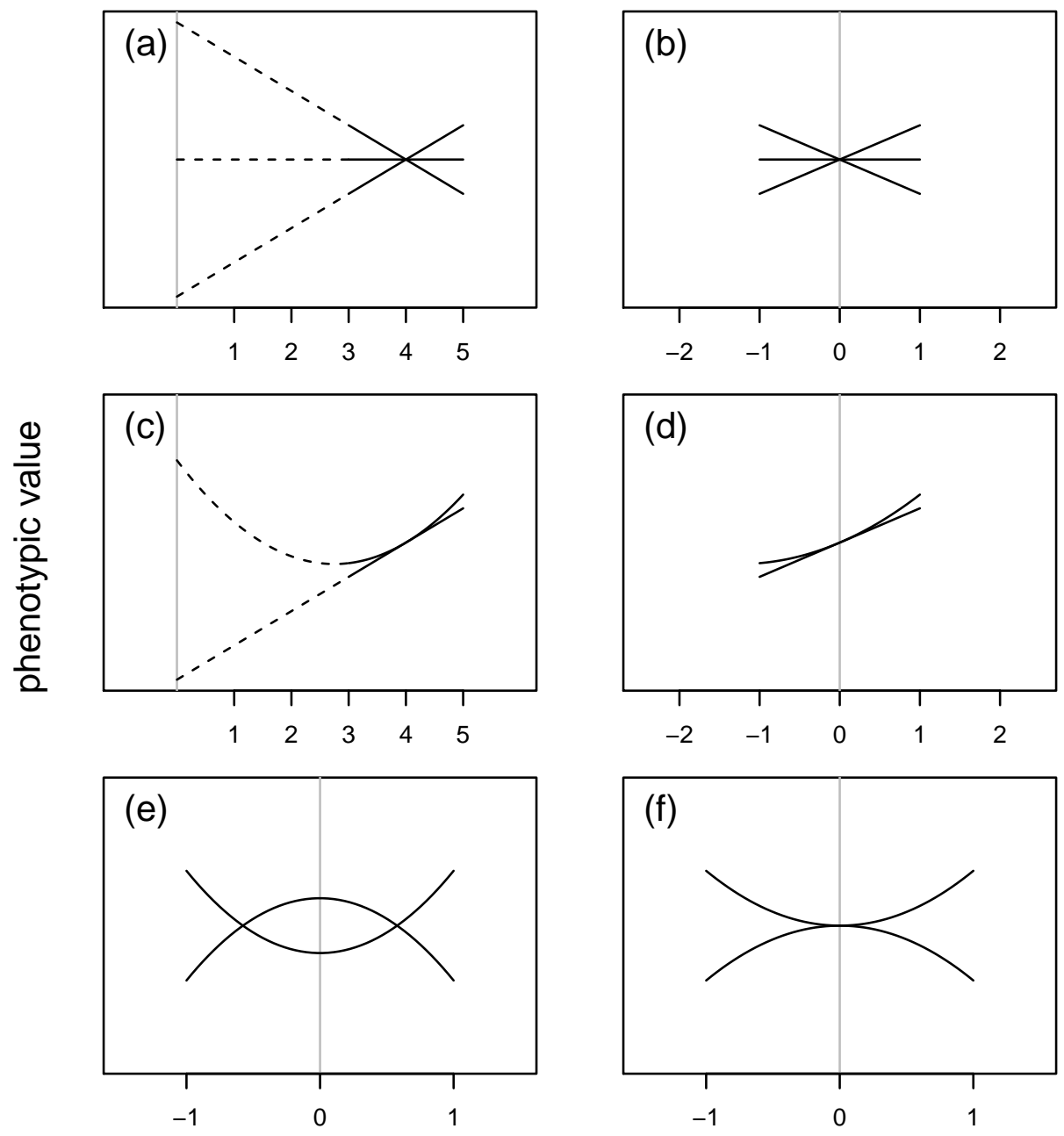

environmental covariate

Figure B1.1: Illustrations of conditions under which mean-centring of an environmental covariate can, and cannot, render regression intercepts interpretable as mean values. See text in Box 1 for details.

730 In quadratic regression analyses of reaction norms, intercepts do not reflect mean values,

731 even under mean-centring, unless there is no curvature. If curvature varies among genetic 732 units, then then differences in intercepts may need not represent differences mean values.

733 Figure B1.1e,f illustrate two alternatives: in part e, intercepts vary between two reaction 734 norms, but mean values do not. In part f, intercepts are identical but mean values differ. 735 This does not mean that quadratic regression analysis of reaction norms cannot provide 736 inference of variation in mean values, only that these values must be derived (see table 3 ). 
737 Box 2: Character-state and reaction norm approaches: the maths

738 Any family of reaction norms, summarised by their mean intercept and slope, and the vari739 ances and covariances of slopes and intercepts, corresponds to a specific set of environment740 specific means and variances of phenotype, and across-environment covariances of phenotype. 741 Expressing a family of reaction norms (or of function-valued phenotypes generally, e.g., de742 velopmental trajectories) in terms of environment-specific (or, for e.g., age-specific in the case 743 of development) means and (co)variances is referred to as a character-state representation. 744 Any complete description of the covariances of parameters of a family of polynomial reaction 745 norms can be translated into what it implies about a character-state representation. The 746 maths involved may initially seem nebulous, but are in fact reasonably straight-forward.

747 The algebraic operation necessary to convert a characterisation of reaction norms to a 748 character state representation is the expression for the variance of a random variable when 749 subjected to a linear transformation. If $x$ is related to $y$ according to $y=b x$, then the variance 750 of $x$ is related to the variance of $y$ according to $\sigma^{2}(y)=b^{2} \sigma^{2}(x)$. If $\mathbf{x}$ and $\mathbf{y}$ are vectors, then 751 a linear transformation might be written $\mathbf{y}=\mathbf{B x}$, where $\mathbf{B}$ is a matrix containing coefficients 752 by which elements of $\mathbf{x}$ are related to elements of $\mathbf{y}$. If instances of $\mathbf{x}$ have (co)variances $\boldsymbol{\Sigma}_{\mathbf{x}}$, 753 then the (co)variances of $\mathbf{y s}$ are given by $\boldsymbol{\Sigma}_{\mathbf{y}}=\mathbf{B} \boldsymbol{\Sigma}_{\mathbf{x}} \mathbf{B}^{T}$, where $\mathbf{B}^{T}$ is the transpose of $\mathbf{B}$ 754 (i.e., a matrix where rows and columns are exchanged).

755 To use the algebra of variances under linear transformation to convert covariances of 756 polynomial coefficients of reaction norms into their character-state representation, we must 757 compose matrices $\mathbf{B}$ that reflect the environments in which we want to express the variances, 758 and among which we might want to know covariances. An example might be most useful at 759 this point. The estimated covariances of intercepts, slopes, and quadratic terms of reaction 
norms of spot number to temperature are

$$
\Sigma_{x}=\left[\begin{array}{ccc}
0.381 & -7.89^{-3} & -8.18^{-3} \\
-7.89^{-3} & 2.35^{-3} & 2.97^{-4} \\
-8.18^{-3} & 2.97^{-4} & 1.83^{-4}
\end{array}\right]
$$

761 The quantities by which reaction norm parameters must be multiplied to give expected 762 phenotype in any given environment are polynomial values corresponding to that environ763 ment's numerical value. In the analyses of Drosophila reaction norms, the temperature data 764 were centred to a mean of $19^{\circ} \mathrm{C}$. So, the values of the polynomial function for, say, 16,19 and $76522^{\circ} \mathrm{C}$ (the middle value and nearly the extremes of the temperature range, see figure 2 ), the 766 polynomials would be $\left[(16-19)^{0}(16-19)^{1}(16-19)^{2}\right],\left[(19-19)^{0}(19-19)^{1}(19-19)^{2}\right]$ 767 and $\left[(22-19)^{0}(22-19)^{1}(22-19)^{2}\right]$. The variances and covariances of the reaction norm 768 functions across the temperatures 16,19 and $22^{\circ} \mathrm{C}$ would then be given by

$$
\left[\begin{array}{ccc}
1 & -3 & 9 \\
1 & 0 & 0 \\
1 & 3 & 9
\end{array}\right]\left[\begin{array}{ccc}
0.381 & -7.89^{-3} & -8.18^{-3} \\
-7.89^{-3} & 2.35^{-3} & 2.97^{-4} \\
-8.18^{-3} & 2.97^{-4} & 1.83^{-4}
\end{array}\right]\left[\begin{array}{ccc}
1 & 1 & 1 \\
-3 & 0 & 3 \\
9 & 0 & 9
\end{array}\right]=\left[\begin{array}{ccc}
0.301 & 0.331 & 0.228 \\
0.331 & 0.381 & 0.284 \\
0.228 & 0.284 & 0.239
\end{array}\right] .
$$

769 This covariance matrix represents the covariances at the genetic level, in this case among770 strains, at which reaction norms are inferred. The high correlations among environments 771 (covariances are positive and similar in magnitude to variances) reflect the character-state of 772 representation of the fact that there is modest variation in slopes and curvatures of reaction 773 norms.

774 In general, a reaction norm approach will use fewer parameters than a character state 775 approach. In the Drosophila example, a description of the strain-level covariance matrix of 776 temperature specific phenotype would require estimation of a matrix with 66 parameters. 777 In this specific example with eight strains, these parameters could not be simultaneously 778 estimated. In fact, any pairwise covariance estimate should be regarded as tenuous given 
779 this level of replication across strains. In contrast, the quadratic reaction norm approach 780 estimates six parameters at the level of strain. One should still keep in mind that its inferences 781 are based the only eight strains, but all the information available is simultaneously used to 782 estimate a model with a more sensible number of parameters. In cases where there is sufficient 783 replication to support both character state and reaction norm approaches, their comparison 784 should be useful. For example, such comparisons could identify ranges of the environmental 785 covariate where a low-dimensional random regression model fits adequately or otherwise. 786 For smaller studies, visual comparison of random regression fits to raw data is probably best 787 (figure 2).

788

789 